\title{
Dialysis peritonitis in a patient with chronic kidney disease and multiple comorbidities
}

\author{
Oana Streinu-Cercel ${ }^{1,2^{*}}$, Anca Streinu-Cercel ${ }^{1,2}$, loana Berciu ${ }^{1,2}$, Alina Cristina Neguț ${ }^{1,2}$, Mihai Săndulescu', \\ Maria Magdalena Moțoi ${ }^{2}$, Adrian Streinu-Cercel ${ }^{1,2}$ \\ From The 9th Edition of the Scientific Days of the National Institute for Infectious Diseases Prof Dr Matei Bals \\ Bucharest, Romania. 23-25 October 2013
}

\section{Background}

Patients with peritoneal catheters are at risk for developing infections with germs with altered antibiotic sensitivity, being classified as Carmeli 3 due to repeated invasive contact with the hospital system. A cloudy peritoneal fluid is oftentimes a sentinel sign of infection and medical and surgical management are generally required to clear infection and prevent subsequent reoccurrences.

\section{Case report}

We report the case of a 70 year-old male patient, with chronic kidney disease under peritoneal dialysis, type II diabetes mellitus, cardiac insufficiency, arterial hypertension, dyslipidemia, bilateral carotid atheromatosis, grade 1 obesity, COPD, and history of repeated peritonitis, including a previous episode of sepsis with MSOF.

The presenting complaint, dating back two weeks, consisted of lower abdominal pain followed by two unformed stools; 12 hours later the dialysis fluid turned cloudy; 24 hours later the patient was admitted to a Nephrology Clinic. In the tenth day of symptomatology, the patient became drowsy and was transferred to our clinic, for infectious diseases management.

Clinical exam at admission revealed: mediocre clinical state, bilateral leg and dorsal hand edema, arterial tension $140 / 90 \mathrm{mmHg}$, pulse $80 \mathrm{bpm}$, abdomen distended through peritoneal dialysis, hepatomegaly.

Lab results showed inflammatory syndrome, slight anemia, nitrogen retention syndrome.

Peritoneal fluid cultures grew Rothia mucilaginosa and the patient mentioned recent dentist treatment with fullmouth prosthetic rehabilitation. We performed a complete dental exam and cultures from: gingival sulcus fluid, dental plaque, tongue; results came out positive for Enterococcus faecalis and Candida spp and peritoneal fluid cultures grew Candida lipolytica. Over the course of hospital admission, the peritoneal count rose to over 4000 cells/ $\mathrm{cmm}$, and the patient presented fever, chills, obnubilation.

Under treatment with meropenem, linezolid, voriconazole and peritoneal instillations with vancomycin, the patient's evolution was favorable.

Due to the isolation of Candida lipolytica, together with the nephrologist and the surgeon, on the $23^{\text {rd }}$ day of evolution a subclavian hemodialysis catheter was placed and on the $25^{\text {th }}$ day, the peritoneal catheter was removed (cultures positive for Candida lipolytica). For the long term management of the kidney disease, a hemodialysis fistula was performed.

\section{Conclusion}

The clinical evolution corroborated the initial suspicion of dialysis peritonitis of mixed etiology, fungal and Rothia spp. Close interdisciplinary collaboration between the infectious disease specialist and the nephrologist is mandatory in order to conduct a proper treatment.

\section{Authors' details}

${ }^{1}$ Carol Davila University of Medicine and Pharmacy, Bucharest, Romania. ${ }^{2}$ National Institute for Infectious Diseases "Prof. Dr. Matei Balş", Bucharest, Romania.

Published: 16 December 2013

doi:10.1186/1471-2334-13-S1-P34

Cite this article as: Streinu-Cercel et al:: Dialysis peritonitis in a patient with chronic kidney disease and multiple comorbidities. BMC Infectious Diseases 2013 13(Suppl 1):P34.

* Correspondence: oanastreinucercel@gmail.com

${ }^{1}$ Carol Davila University of Medicine and Pharmacy, Bucharest, Romania

Full list of author information is available at the end of the article 